\title{
A BALLOON TRAJECTORY PREDICTION SYSTEM
}

\author{
I. Musso ${ }^{1}$, A. Cardillo ${ }^{1}$, O. Cosentino ${ }^{2}$, A. Memmo ${ }^{3}$ \\ ${ }^{1}$ Cnuce-Cnr, National Research Council, via Moruzzi 1, Pisa, Italy \\ ${ }^{2}$ Italian Space Agency, Stratospheric Balloons Base, Trapani Milo, Italy \\ ${ }^{3}$ CETEMPS, Center for the Forecast of Severe Weather, Via Vetoio, località Coppito (AQ), Italy
}

\begin{abstract}
Safety requirements and the increase in balloon flight duration make a computerized balloon control system essential. Starting with a global thermodynamic model of a stratospheric balloon (NASA-SINBAD) and 48-hour wind forecast data, a flexible system can be built to predict its trajectory. In order to increase the trajectory forecast accuracy, the thermodynamic model of SINBAD, related to infrared radiation and albedo, has been improved. The model and the methodology have been evaluated by comparing the altitude excursion of some already flown zeropressure balloons, with the altitude excursion computed by SINBAD; meteorological and satellite data (METEOSAT) and meteorological forecast data have been used as input. This system, connected during the mission to the balloon's managing unit, will continuously update the forecast trajectory and will enable real and simulated data to be compared. In this way it will also be possible to simulate the balloon flight trajectory in case of any failures. This paper explains the solution adopted for this system and the application that was carried out for the Italian Space Agency's 2002 summer balloon campaign.
\end{abstract}

\section{INTRODUCTION}

Managing a balloon's flight entails being able to predict its trajectory and altitude variations. Forecast, sounding and satellite data and an efficient thermodynamic model of the balloon are necessary. The prediction has to be done in two different cases: several hours before the flight and in real time during it. In the first case there may be up to 48 hours between the preflight analysis and the launch, so forecast data are essential. In this premission phase, the trajectory prediction is used for checking the preliminary compliance with the flight constrains (safety, altitude, over-fly of target areas, rendezvous...). In the second case the simulation aims to reduce the error of the preflight analysis, on the basis of up-to-date data, and to reduce the time necessary to take operational decisions. The altitude-time function of the balloon, since the last moment before the analysis, will be taken into account; the simulation that best trace it will be chosen and projected into the future. This method is important during the ascending phase when the exact free lift of the balloon is not known and/or when a particular cyclonic condition is expected, without the possibility to evaluate the exact amount of the infrared radiation and the albedo of the clouds. Particular cases can also then be inserted and evaluated during the flight, such as possible failure (gas loss) and maneuvers (ballast discharge, valve openings..).

\section{FORECAST ATMOSPHERIC MODEL}

To improve the balloon's trajectory prediction different mesh grids of atmospheric data have been used and combined in an appropriate way. In particular, we have adopted the Fifth-generation Mesoscale Model (MM5) version 3, developed by the Pennsylvania State University/National Center for Atmospheric Research, in the following configuration:

- Non-hydrostatic run (Grell et al. 1994, Dudhia 1993);

- Kain-Fritsch (Kain and Fritsch, 1993) convective parameterization scheme;

- MRF parameterization of boundary layer (Troen et al. 1986); 
- Lambert conformal projection;

- two nested domains: the first one with $27 \mathrm{~km}$ grid step in the area lat $[33,48]$ lon $[-11,23]$, the second one with $9 \mathrm{~km}$ grid step in the area lat $[35,39]$ lon $[10,17]$;

- 30 alpha-sigma levels;

- top pressure level: 10 mbar;

- Model output: 1 hour time step;

- Model initialized with data from the National Centers for Environmental Prediction.

The MM5 output was used to predict the ascending trajectory of the balloon and the descending trajectory of the parachute. In addition, the higher resolution of the second domain allowed us to consider with good approximation the complex orography and boundary layer conditions in order to have a better trajectory prediction (Figure 1.).

To compute the trajectory for pressures smaller than $10 \mathrm{mbar}$, data from the European Centre for MediumRange Weather Forecasts have been used, with a 6-hour time step, 0.5 degree grid step (about $50 \mathrm{~km}$ at the considered latitudes) and six vertical pressure levels. If we consider that in the stratosphere there is no great change in the wind components for several hours and that the wind remains regular enough for some hundred kilometers, then these data represent, in this case too, a good wind component approximation to trace the stratospheric flight simulation (Figure 2.).

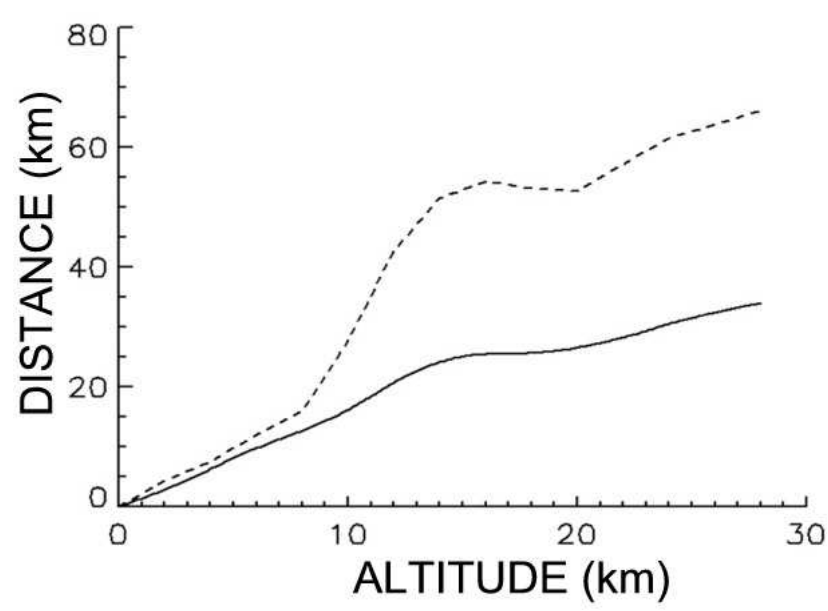

Fig. 1. Distance between sounding and 24-36 hour forecast simulations for ascending altitude levels. Maximum (dashed line) and average (solid line) during the month of July 2002.

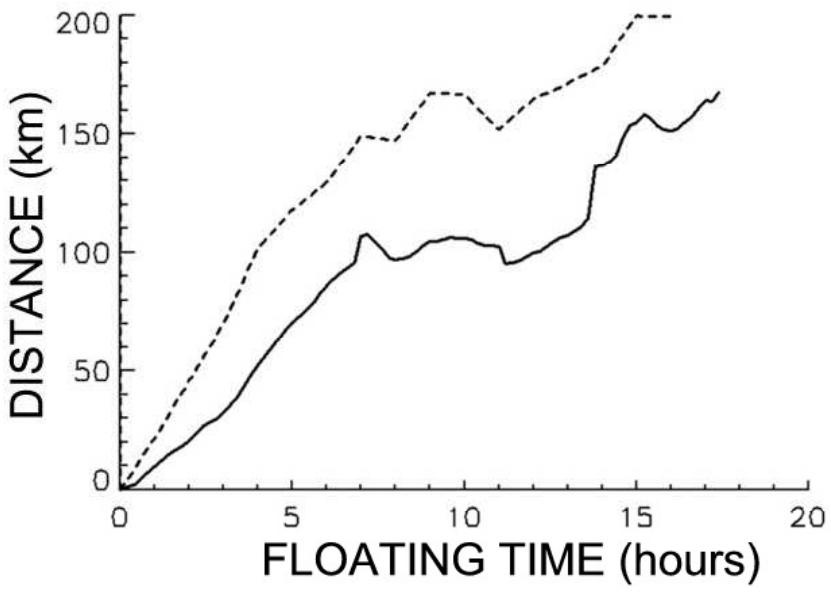

Fig. 2. Distance between stratospheric balloon floating trajectories and 24-36 hour forecast simulations for elapsed time. Maximum (dashed line) and average (solid line) during the 2001 and 2002 campaigns

\section{THERMODYNAMIC BALLOON MODEL}

The adopted thermodynamic model comes from the NASA-SINBAD software where the balloon is drawn like a spherical homogeneous gas bubble. The total infrared radiation is evaluated using the average ground temperature, measured or expected. A preliminary test was made comparing the model with respect to some past flights with diurnal and nocturnal ascending phases.

\section{Ascending phase}

At the launch (Figure 1, phase A) the initial velocity depends essentially on the free lift, sun and infrared radiation. Another effect comes from the difference between air and initial mean gas temperatures, which depend on the inflation conditions. Below $30 \mathrm{~km}$ in altitude the most significant heat flux is the convection between air and the balloon's skin (Figure 1, phase B). In particular the extension of the tropopause and its lowest temperature are very important in order to evaluate the time necessary to reach the floating altitude. Above $30 \mathrm{~km}$ a correct evaluation of the infrared and solar radiation becomes fundamental (Figure 1, phase C), in particular the infrared background which depends on the ground or cloud's top temperature. The convection in this phase is small due to the low density of the air. 
The simulator was and will be applied to some real flights with known free lift, known ground temperature and cloud cover for nocturnal and diurnal ascending phases. This study permits the software parameters to be modeled on the basis of experimental data and SINBAD to be used on Transmediterranean flights.

The drag coefficient can change the result of the simulation in particular during the initial phases, when a relatively high velocity is expected. It has a small effect above $20 \mathrm{~km}$ where the density of the air starts to decrease significantly. A good approximation of the analyzed flights is obtained using a constant drag coefficient of 0,45 in accordance with the sphere model.

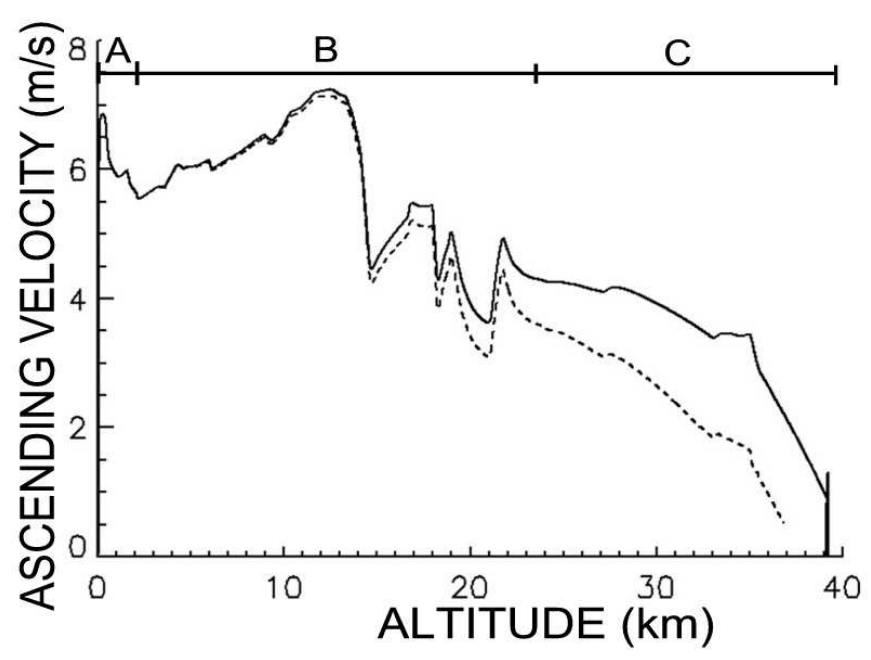

Fig. 3. Example of ascending velocity function for low (dashed lines) and high infrared background (solid line)

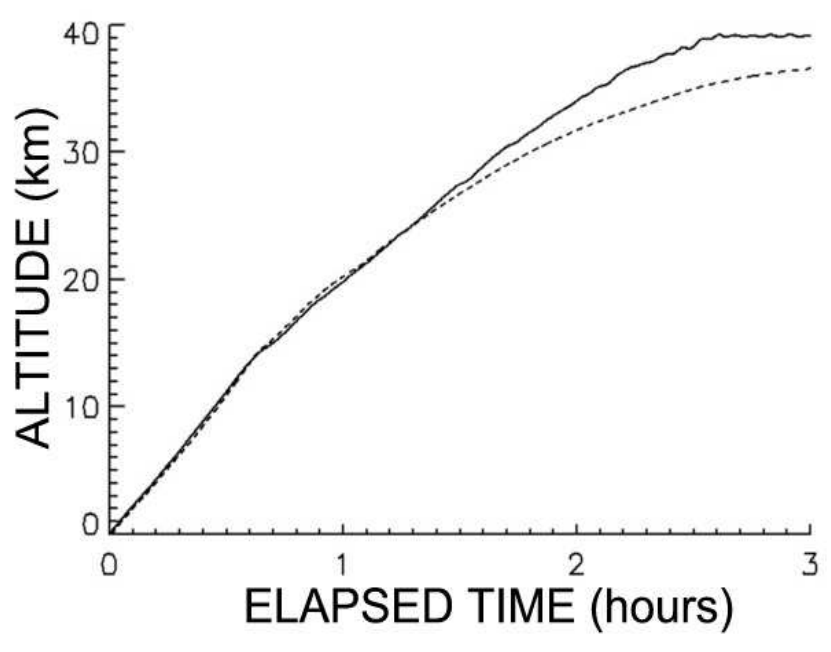

Fig. 4. Ascending phase for a nocturnal flight (solid line) and his simulation (dashed lines) when a low infrared background is imposed

For a correct simulation it is very important to pay attention to a few particular points:

- Starting free lift: the exact amount of gas cannot be evaluated directly at the launch, so is important to take this possible variation into account.

- Air temperature: a sudden variation in the air's temperature produces a similar variation in the ascending velocity, in particular before and during the tropopause.

- Infrared background: an error on this evaluation can affect the predicted time necessary to reach floating altitude. This is fundamental for nocturnal ascending phases above $30 \mathrm{~km}$ altitude, when the infrared radiation is the most important heat flux (Figure 4).

From a simulation it is important to evaluate:

- Duration of the ascending phase: this is important when a scientist's requirement is to reach the floating altitude in a particular time, or when it is important to keep the ascending trajectory under control for safety reasons.

- Gas loss: comparing simulations with real data during the flight, makes it possible to understand if a gas loss is in progress and then to find an updated trajectory.

\section{Floating phase}

Similar remarks can be made for the floating phase. Here the infrared background and the albedo are important for the determination of the minimum altitude reached during the night-day cycle. Air temperature doesn't have a great effect because the balloon usually flies above thirty kilometers where the convection is low. The prediction of the altitude-time function, during the floating phase, is important for obtaining a correct trajectory and because some scientists usually impose constrains for particular types of experiments. Essentially it is necessary to evaluate how much ballast must be dropped in order to keep a certain minimum flight altitude.

By evaluating satellite images in the infrared and visible channels it is possible to calculate the infrared background and albedo that depend on land and/or cloud positions and types. These are real data, but are only important for post-flight analysis or real-time low duration simulations: the time during which the image can be considered valid. For long duration or pre-flight simulations, forecast information is again necessary. The following 
simplified method has been used: where the relative humidity reaches the limit of condensation a cloud is considered to be present and its top temperature is approximated with the air temperature at the same altitude. The albedo is obtained as a linear function of the cloud height. For clear skies a brightness temperature is chosen equal to the air temperature at ground level and the albedo, the mean value for land $(0,15-0,2)$.

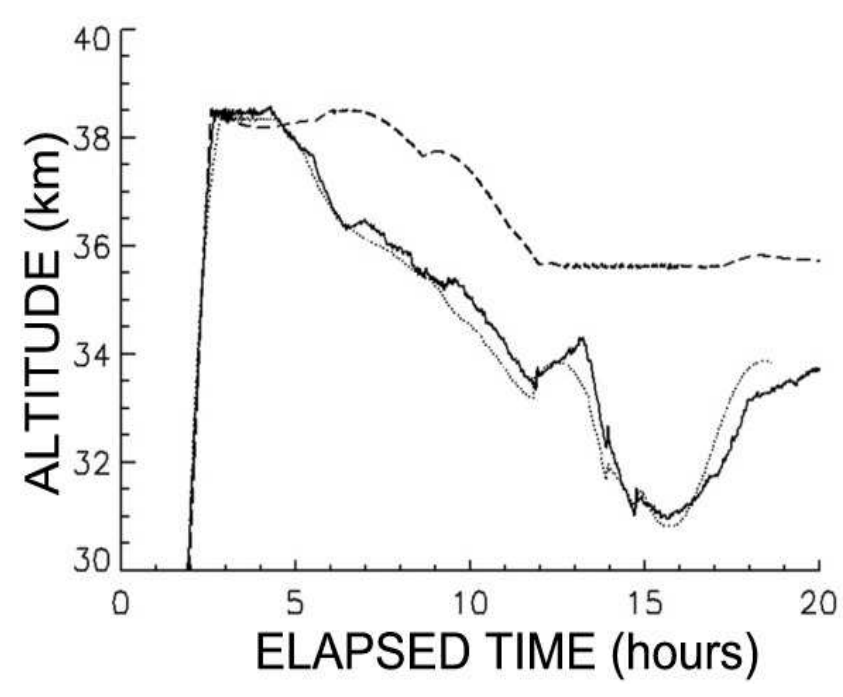

Fig. 5. Example of balloon flight with bad meteorological conditions (solid line) and its simulations with measured (dot) and predicted infrared background and albedo (dashed lines)

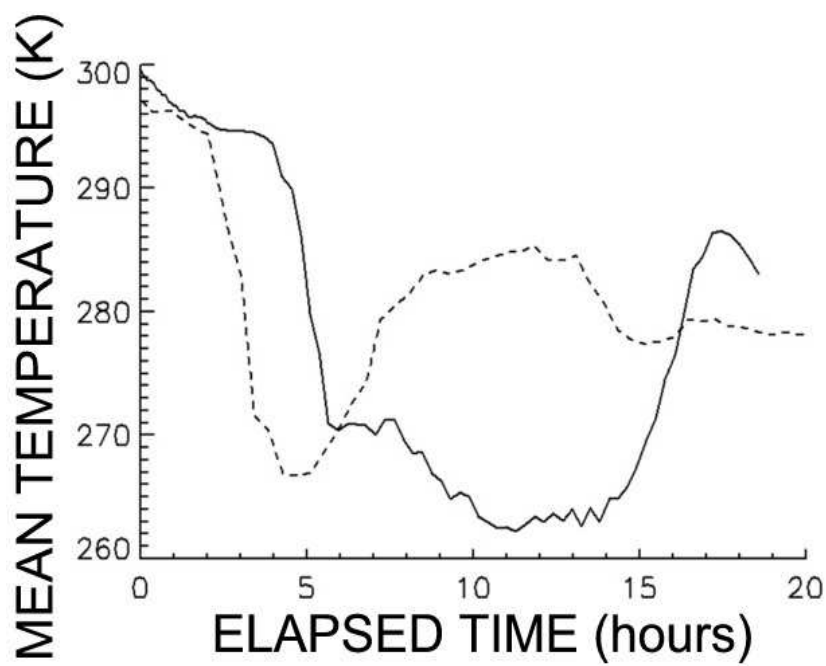

Fig. 6. Mean brightness temperature, seen by the balloon, for Figure 5 flight. Measured values (solid line, "Eumetsat Archive") and 24-36 hour forecast (dashed lines)

When a cyclonic condition is expected it is important try to evaluate some parameters:

- When the balloon will meet the cloud area;

- How long this condition will last;

- What the mean brightness temperature will be during this period.

Figures 5 and Figure 6 show an example of flight with bad meteorological conditions. In this case the cyclonic period started two hours after the predicted one and its mean temperature was about $270 \mathrm{~K}$ compared to $280 \mathrm{~K}$. This difference gives an excursion of four kilometers between real and predicted minimum altitudes.

\section{SOFTWARE STRUCTURE}

The adopted structure is shown in Figure 7. Block 1 is Trackball, the software already available (O. Cosentino, A. Cardillo et al 2001) which receives the simulations. This architecture is important to maintain software reusability and stability; this means that block 1 can be used on its own as well. The simulations are superimposed onto cartography, and satellite images or real flights onto different types of graphs. There are two main uses: pre-flight analysis and real-time simulations.

\section{Preflight analysis}

Starting with the balloon's characteristics, payload weight, sounding and forecast data, preliminary simulations are carried out. The atmospheric data used are soundings and/or forecast, for the ascending phase, and forecast for the subsequent floating phase. The simulations are made using the maximum and minimum expected free lifts at launch and predicted cloud covers; finally the wind dispersions are applied for each trajectory (Figure 1 and Figure 2). For each point along the trajectory a predicted impact area is then calculated using forecast data and the parachute's characteristics. The result is a conic shaped 3d-surface which includes all simulations and which will contain the balloon's trajectory, if nominal conditions are respected. In addition a polygon will be identified on the ground, which contains all possible landing points (I. Musso, A. Cardillo et al 2002). Now it is possible to evaluate a safety flight parameter by projecting the surfaces on a map where an assessment index is shown. Finally 
the altitude-time functions of the obtained simulations are collected in a file: they will serve as an archive of what may happen during the flight and which will be used for the real-time operations.

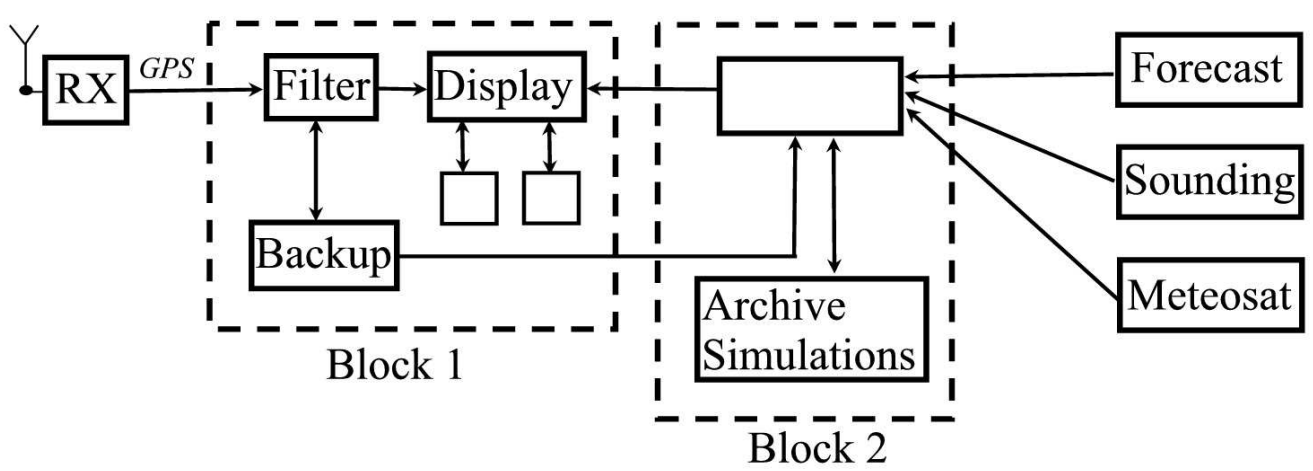

Figure 7. Structure adopted for the preflight and real-time balloon trajectory simulator

It is important to stress that sounding data may give rise to minor errors, with respect to forecast data, if the time between sounding and launching is less than six hours. If the opposite occurs, the wind dispersions may exceed that shown in Figure 1. The launch procedure for a big balloon takes about three hours (balloon preparation, telemetry checks and inflation...) and the sounding about two hours. This means that the decision to launch usually has to be taken using forecast data.

\section{Real-time simulations}

During the flight, Trackball (block 1 in Figure 7) uses a disk backup archive in case the computer crashes, in order to restart the session where it has just left off. This archive can be consulted to obtain the current trajectory of the balloon. The second block (Figure 7) compares its simulations with real data, it finds the one that best traces the altitude-time graph and it projects this simulation into the future. Only one trajectory is now obtained for which the wind dispersion needs to be applied. The results are new conic and land surfaces starting from the balloon's current position. They will be shown by Trackball with the real trajectory and superimposed using additional layers.

The simulations of the altitude-time function can be chosen from the ones done before the flight using the expected conditions. In real-time a simple Newton-Raphson routine finds the best solution with linear interpolation between the two nearest simulations. It takes less time compared to calculating a new one from the current point. In this way, for example, an ascending altitude-time function with a $10 \%$ free lift can be obtained from those with $8 \%$ and $12 \%$. If up-to-date data are available, sounding forecast or infrared satellite images, or if something unpredicted happens, such as gas loss or maneuvers, new simulations must be carried out by evaluating the balloon's current configuration.

In the recovering phase Trackball is able to predict the parachute trajectory but it needs up-to -date atmospheric data. For this purpose, every few minutes the second software program finds the information from sounding or forecast archives and sends the necessary data to Trackball. The sounding archive plays an important part in this application. The latest data for the flight zone have to be collected and the best one chosen in correlation with the balloon's position and time. Alternatively forecast data should be used when the best sounding is too far away in time or space. This part is currently under implementation.

Another use of the new software is to supply land or meteorological satellite images centered on the balloon's current position. In this way it is possible to find the most recent one from an archive or to give only a part of a high-resolution image without creating extra work for Trackball. 


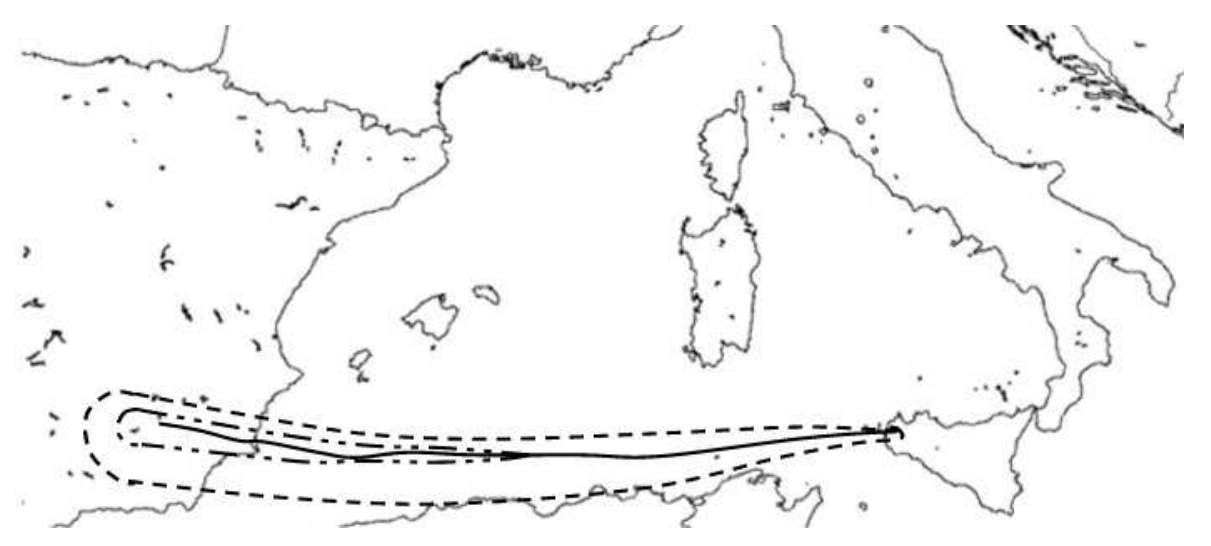

Fig. 8. Example of preflight (dashed line) and mid-flight (dashed-dotted) simulations for a balloon flight (solid line)

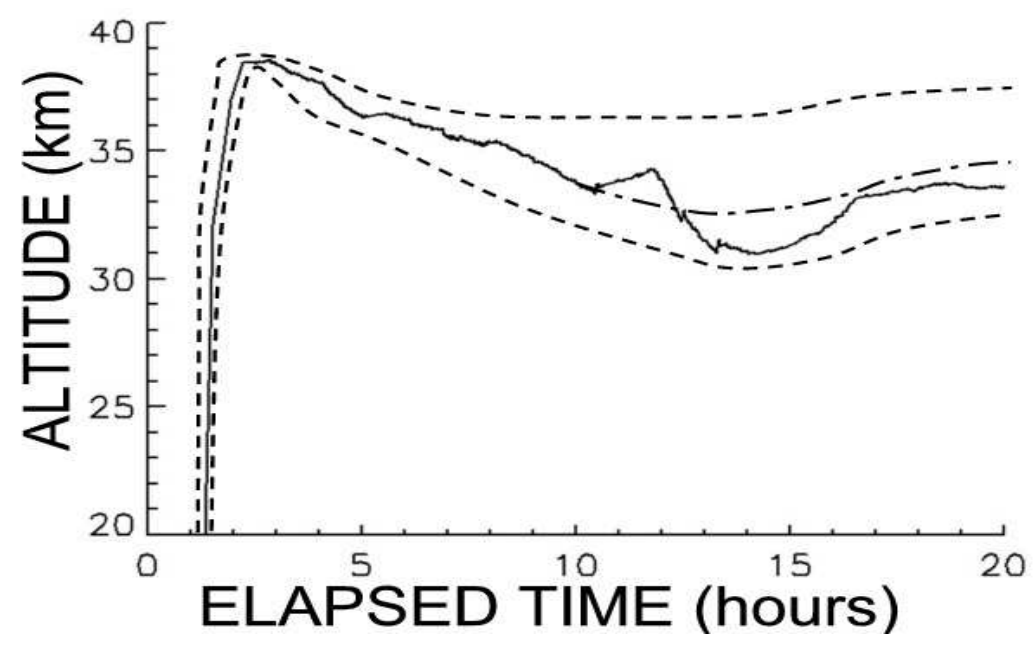

Fig. 9. Extreme simulations for preflight analysis (dashed lines) and mid-flight interpolation (dashed lines-dot)

\section{REFERENCES}

Cosentino, O., A. Cardillo et al., A software development plan for manage a stratospheric balloons flight, presented at the European Rocket and Balloon 15, Biarritz, France, May 2001.

Dudhia, J., A non hydrostatic version of the Penn State-NCAR mesoscale model: Validation tests and Simulation of an Atlantic Cyclone and cold front. 1993, Mon.Wea. Rev., 121, 1493-1513.

Grell, G. A., Dudhia J. and D.R. Srauffer, A Description of the fifth-Generation Penn State/NCAR Mesoscale Model (MM5). 1994, Tech. Note NCAR/Tn-398+STR.

Kain J. S., Fritsch J. M., Convective Parameterization for mesoscale model: the Kain-Fritsch. 1993, The representation of cumulus convection in numerical models, K. A. Emanuela and D.J. Raymond, Eds., Amer. Meteor. Soc., 246

Morris, A. L., Scientific Ballooning Handbook, NGAR Technical Note, Boulder, Colorado, USA, 1972

Musso, I., A. Cardillo, O. Cosentino, The Hasi flight control strategy, presented at ISTS 23, Matsue, Japan, May 2002.

Phulpin, T., S. Venel et al, Models and instrumentation for monitoring the thermal behaviour of stratospheric balloons, presented at European Rocket and Balloon 15, Biarritz, France, May 2001

Raqué, S. M., Sinbad User's Manual, NASA Balloon Projects Branch, Wallops Island, VA, USA, 1993

Troen, I. And Marth L., A Simple Model of atmospheric boundary layer: sensivity to surface evaporation. 1986, Boun. Layer Mereor., 37, 129-148.

E-mail addresses: $\quad$ ivano.musso@guest.cnuce.cnr.it ; andrea.cardillo@cnuce.cnr.it orazio.cosentino@asi.it ; adelaide.memmo@pstabruzzo.it 